\title{
A SURVEY OF BOUNDED SURGERY THEORY AND APPLICATIONS
}

\author{
STEVEN C. FERRY, IAN HAMBLETON, AND ERIK K. PEDERSEN
}

\section{INTRODUCTION}

We begin by attempting to answer the question: What is bounded topology and why do people study it?

In his 1963 paper [13], Connell defines a homeomorphism $h: \mathbb{R}^{n} \rightarrow \mathbb{R}^{n}$ to be bounded if there is a $k>0$ such that $\|h(x)-x\|<k$ for all $x \in \mathbb{R}^{n}$. He notes that if $\varphi: \mathbb{R}^{n} \rightarrow$ int $D^{n}$ is a radial homeomorphism, then $\varphi \circ h \circ \varphi^{-1}:$ int $D^{n} \rightarrow$ int $D^{n}$ extends by the identity on $\mathbb{R}^{n}-$ int $D^{n}$ to a homeomorphism from $\mathbb{R}^{n}$ to itself. It follows easily via the Alexander Trick that bounded homeomorphisms are isotopic to the identity and that bounded homeomorphisms are stable, that is, that every bounded homeomorphism $h$ can be written as a composition $h_{k} \circ \cdots \circ h_{1}$ of homeomorphisms $h_{i}: \mathbb{R}^{n} \rightarrow \mathbb{R}^{n}$ such that $h_{i} \mid U_{i}=i d$ for some nonempty open set $U_{i} \subset \mathbb{R}^{n}$. Connell goes on to prove by a controlled engulfing argument that every stable self homeomorphism of $\mathbb{R}^{n}$ can be approximated arbitrarily closely by PL homeomorphisms. In [14] Connell and Hollingsworth set up a parallel algebraic theory of geometric groups.

Such questions were of interest because of their close relation to the annulus conjecture and the triangulation of manifolds. It was known [5] that the annulus conjecture is true in all dimensions if and only if for all $n$ every orientation-preserving homeomorphism of $\mathbb{R}^{n}$ is stable. It was also known that a counterexample to the annulus conjecture would give a nontriangulable manifold with boundary and that a strong relative theorem approximating homeomorphisms by PL homeomorphisms would imply that all manifolds were triangulable.

Edwards and Kirby [16] used bounded techniques to prove the local contractibility of the homeomorphism groups of topological manifolds. The basic idea for a homeomorphism $h: \mathbb{R}^{n} \rightarrow \mathbb{R}^{n}$ is to show that if $h$ is sufficiently close to the identity on the unit ball, then the restriction of $h$ to a smaller ball can be extended to a self-homeomorphism of $T^{n}$. Passing to the universal cover gives a bounded homeomorphism of $\mathbb{R}^{n}$ which agrees with the original homeomorphism on a neighborhood of the origin, showing that the original homeomorphism is stable. A pair of Alexander tricks supplies an isotopy from the original homeomorphism to the identity. Injecting surgery theory - via the PL classification of homotopy torii - into a variant of this argument proves that all orientation-preserving homeomorphisms of $R^{n}$ are stable for $n \geq 5$ and that the annulus conjecture is true in dimensions $\geq 5$. This combination of torus arguments with surgery theory proved to be very powerful, and by the early 1970's

Partially supported by National Science Foundation Grant DMS 9003746. 
had led to the Kirby-Siebenmann theory of combinatorial triangulations [36], Siebenmann's CE approximation theorem [54], Chapman's proof of the topological invariance of Whitehead torsion [9], and West's proof that every compact ANR has the homotopy type of a finite polyhedron [60].

By 1976, Ferry [19] had used torus techniques to generalize Chapman's theorem on topological invariance of torsion, showing that sufficiently controlled homotopy equivalences between finite polyhedra are simple. Chapman and Ferry [12] then proved a similar generalization of Siebenmann's CE approximation theorem, showing that controlled homotopy equivalences between high-dimensional topological manifolds can be approximated by homeomorphisms. This gave applications to topological embeddings [20], for example one easily shows that if an embedding $i: S^{n-1} \rightarrow S^{n}$ is 1-LCC on one side, then $i\left(S^{n-1}\right)$ bounds a ball on that side. Farrell and Hsiang [18] used these controlled vanishing results in their solution of the topological Euclidean space form problem. Anderson and Hsiang [2] used bounded geometry and torus tricks in their study of homeomorphisms of stratified spaces and the obstructions to triangulating locally triangulable stratified spaces. This resulted in an obstruction theory and the first appearance of Bass' $K_{-i}$ groups as obstructions in geometric problems. The AndersonHsiang paper also contained the basic observation that the space of self-homeomorphisms of $M * S^{n}$ relative to $S^{n}, \mathrm{M}$ a manifold, is homotopy equivalent to the space of bounded homeomorphisms of $M \times \mathbb{R}^{n+1}$, boundedness measured in $\mathbb{R}^{n+1}$. Together with the isotopy extension theorem, this allows the analysis of stratum-preserving homeomorphisms of twostratum spaces. This approach carries over nicely to the study of group actions, see e. g. $[4,29]$.

In the late 1970's and early 1980's Quinn proved his celebrated End Theorem and used controlled techniques to prove the Resolution Theorem, the Obstruction to Resolution, and the Annulus Conjecture in Dimension 4 [47, 48, 49, 50]. Bounded topology plays a role in Quinn's work - his algebraic torus trick is the source of the basic vanishing theorem - but in the main his method is to use the Connell-Hollingsworth geometric group apparatus, the utility of which is greatly improved by Quinn's stability theorem [48] which shows that under reasonable hypotheses, $\epsilon$-Whitehead groups are independent of $\epsilon$ for $\epsilon$ small. A geometric version of this theorem was proved by Chapman [10] at about the same time. Quinn's methods sufficed for the construction of an obstruction theory and for the the solution of a number of outstanding problems, but there are difficulties in developing a satisfactory and computable algebraic theory based on this approach. To quote Quinn: "Category theory does not take gracefully to the addition of $\epsilon$ 's," the problem being that arbitrary compositions of $\epsilon$-controlled maps can be arbitrarily large and that the control afforded by stability does not overcome all of the resulting problems.

By contrast, arbitrary compositions of bounded maps are bounded. This categoricity means that one can construct categorical algebraic versions of bounded theories. It turns out that the resulting theory is a deloop of the geometric group theory when the latter makes 
sense. The early work along these lines is due to Pedersen [40], [41], [42] and PedersenWeibel [43], [44] and was further developed by Anderson and Munkholm in [3]. As observed by Anderson and Hsiang, problems involving stratified spaces fit very naturally into the bounded machinery. This is well illustrated by the work of Hambleton and Pedersen on cocompact group actions on $S^{k} \times \mathbb{R}^{n}$ described in Sections 6 and 7. Bounded surgery turns out to be sufficient for deriving Quinn's obstruction to resolution. We describe the approach in Section 5. Using extensions of Quinn's stability theorem, the theory also applies to some problems which look like " $\epsilon$-problems" rather than bounded problems. This is illustrated in Sections 8 and 9 where we describe the use of bounded methods to prove an improved stability theorem and count simple homotopy types in Gromov-Hausdorff space.

The object of this introduction is to motivate the study of bounded topology, not to give a historical survey of the entire area. We should however mention Carlsson's usage of bounded algebra in his approach to the K-theory version of the Borel conjecture [8], FerryWeinberger's use [24] of bounded topology in work on the Novikov conjecture, Hughes work on approximate fibrations [33, 34], Weiss-Williams study of the space of homeomorphisms of manifolds [58, 59], using among other bounded techniques, and Ranicki's development of the algebraic side of bounded L-theory, including the original definition of the $L^{-i}$-groups $[51,52,53]$. We should also mention, that there are controlled and/or stratified surgery theories due to Hughes-Taylor-Williams [35], and Weinberger [57].

\section{BOUNDED CW THEORY}

Definition 2.1. A map $p: X \rightarrow M$ from a topological space $X$ to a metric space $M$ is eventually continuous if there exists $k>0$ and an open covering $\left\{U_{\alpha}\right\}$ of $X$ so that the diameter of $p\left(U_{\alpha}\right)$ is smaller than $k$. It is also required that the inverse image of a bounded set be relatively compact.

Definition 2.2. A map of metric spaces $f: M_{1} \rightarrow M_{2}$ is eventual Lipschitz if there exists $k$ and $l$ so that $d(f(x), f(y))<k d(x, y)+l$ and the inverse image of a bounded set is bounded. Two such maps are homotopic if they are a bounded distance apart. An eventual Lipschitz homotopy equivalence is a quasi-isometry in the sense of Gromov.

Example 2.3. Let $K$ be a finite complex and choose a generating set for $\pi=\pi_{1}(K)$. The group $\pi$ may be thought of as a metric space with the word metric. Define a map $p: \widetilde{K} \rightarrow \pi$ as follows: For every open cell of $K$ choose a lift in $\widetilde{K}$. Send this open cell of $\widetilde{K}$ constantly to $1 \in \pi$ and extend to translates of the open cell in $\widetilde{K}$ by equivariance. The map $p$ is clearly not continuous but it is eventually continuous since $K$ only has finitely many cells. Choosing different lifts of the cells of $K$ produces a different map, say $q$, but the distance between $p$ and $q$ is bounded. The choice of generators for $\pi$ does affect the metric of $\pi$ but only up to quasi-isometry.

Definition 2.4. Given $p: X \rightarrow M$ an eventually continuous map, we say that a (continuous map) $f: Y \rightarrow X$ is a bounded homotopy equivalence if there is a map $g: X \rightarrow Y$ and 
homotopies $F: g \circ f \sim 1_{Y}$ and $G: f \circ g \sim 1_{X}$ so that the diameters of $p f F(x \times I)$ and $p(G(y \times I))$ are uniformly bounded (independent of $x$ and $y$ ).

Definition 2.5. A bounded $C W$ complex $p: X \rightarrow M$ consists of a CW complex $X$ and an eventually continuous map $p$ so that the diameters of $p$ applied to a cell is uniformly bounded.

Definition 2.6. A metric space is boundedly contractible if for every $k$ there exists $\ell$ so that a subset of diameter smaller than $k$ contracts in a subset of diameter smaller than $\ell$.

Example 2.7. Euclidean space $\mathbb{R}^{n}$ and hyperbolic space $\mathbb{H}^{n}$ are clearly boundedly contractible. In general if $K$ is a finite $K(\pi, 1)$ and $\widetilde{K}$ is given an induced metric ( $\pi$ acts by isometries), then $\widetilde{K}$ is boundedly contractible because every set of diameter $k$ is contained in a translate of the unit ball of diameter $l$ for some $l$ ).

Example 2.8. The subset $0 \times[0,1] \cup[0, \infty) \times 0 \cup[1, \infty) \times 1$ of $\mathbb{R}^{2}$ is not boundedly contractible but it is homeomorphic to $\mathbb{R}$.

The purpose of introducing the concept "eventually continuous" is to be able to disregard local phenomena in the metric space. To comfort the reader there is the obvious.

Proposition 2.9. If $p: X \rightarrow M$ is a finite dimensional bounded $C W$ complex and $M$ is boundedly contractible, then there is a continuous map $q: X \rightarrow M$ so that $d(p(x), q(x))$ is uniformly bounded.

Definition 2.10. A bounded CW complex $X \rightarrow M$ is -1 -connected if there is a $k>0$ so that for each $m \in M$ there is an $x \in X$ with $d(p(x), m)<k$.

Definition 2.11. A bounded CW complex is 0-connected if for every $k>0$ there exists $\ell>0$ such that for all $x, y \in X: d(p(x), p(y))<k$ implies there exists a path $\alpha: I \rightarrow X$ from $x$ to $y$ so that $\operatorname{diam}(\alpha(I))<\ell$.

Definition 2.12. A bounded CW complex is 1-connected if for every $k>0$ there is $\ell>0$ so that a loop $\alpha: S^{1} \rightarrow K$ with $\operatorname{diam}\left(p\left(\alpha\left(S^{1}\right)\right)<k\right.$ bounds a disc $A: D^{2} \rightarrow K$ with $\operatorname{diam}\left(p\left(A\left(D^{2}\right)\right)<\ell\right.$.

Definition 2.13. A bounded CW complex $X \rightarrow M$ has a bounded fundamental group $\pi=\pi_{1} X$ if the universal cover $\widetilde{X} \rightarrow M$ is 0 and 1-connected.

Definition 2.14. A free bounded $G$-CW complex $X \rightarrow M$ consists of a bounded CWcomplex together with a free cellular action of $G$ on $X$ and an action by quasi-isometries on $M$ making $p$ equivariant.

Example 2.15. If $X \rightarrow M$ has bounded fundamental group $\pi$, then $\tilde{X} \rightarrow M$ is a free bounded $\pi$-CW complex with the trivial action on $M$ 


\section{Bounded Algebra}

Definition 3.1. Given a ring $R$, and a metric space $M$, the category $\mathcal{C}_{M}(R)$ has objects $A$, a collection $\left\{A_{x}\right\}_{x \in M}$ where $A_{x}$ is a finitely generated free R-module, such that

$$
\left\{x \mid A_{x} \neq 0\right\} \text { is locally finite in } M \text {. }
$$

A morphism $\varphi: A \rightarrow B$ is a collection of morphisms

$$
\varphi_{y}^{x}: A_{x} \rightarrow B_{y} \text { satisfying }
$$

there exists $k=k(\varphi)$ so that

$$
\varphi_{y}^{x}=0 \text { if } d(x, y)>k
$$

Composition is given by

$$
(\varphi \circ \psi)_{y}^{x}=\sum_{z \in M} \varphi_{y}^{z} \circ \psi_{z}^{x}
$$

the sum being finite by the conditions.

In the case

$$
M=O(K)=\left\{t \cdot \underline{x} \in \mathbb{R}^{N+1} \mid t \in[0, \infty) \quad \underline{x} \in K \subseteq S^{N}\right\}
$$

$K$ is finite complex contained in a sphere, the K-theory of $\mathcal{C}_{O(K)}(R)$ is understood.

Theorem 3.2. ([44])

$$
K_{*}\left(\mathcal{C}_{O(K)}(R)\right) \cong h_{*-1}(K, A \lg K R)
$$

where the right hand side is the generalized homology theory with spectrum the non-connective algebraic K-theory spectrum of the ring $R$.

Remark 3.3. For $K$ a sphere, this theorem says $K_{1}\left(\mathcal{C}_{\mathbb{R}^{n}}(R)\right) \cong K_{1-n}(R)$. It is possible to give a direct elementary proof that the functor $K \rightarrow K_{i}\left(\mathcal{C}_{O(K)}(R)\right)$ is half exact when $i \leq 2$. This suffices in principle to compute the obstruction groups in Quinn tame ends [48], epsilon h-cobordisms etc.

Given a bounded CW complex $X \rightarrow M$, the cellular chains are denoted $D_{\#}(X)$ when they are thought of as a chain complex in the category $\mathcal{C}_{M}(\mathbb{Z})$. The following theorem follows from [3] in the case where the reference map is continuous.

Theorem 3.4. Suppose $Y \stackrel{f}{\rightarrow} X$ is a map of $-1,0,1$ connected bounded $C W$ complex ( $p \circ f$ is the reference map for $Y$ ). Then $f$ is a bounded homotopy equivalence if and only if

$$
F_{\#}: D_{\#}(Y) \rightarrow D_{\#}(X)
$$

is a chain homotopy equivalence in $\mathcal{C}_{M}(\mathbb{Z})$. 
Suppose $R$ is a ring with involution. We then define an involution (in the sense of Ranicki [52]) on the category $\mathcal{C}_{M}(R)$ by $\left\{A^{*}\right\}_{x}=\left\{A_{x}^{*}\right\}$ (pointwise involution). Given a bounded CW complex $X \rightarrow M$. We define the cochains of $X, D^{\#}(X)$ to be $\left(D_{\#}(X)\right)^{*}$. The direct sum and direct product functors from $\mathcal{C}_{M}(R)$ to the category of $R$ modules induce functors of chain complexes. It is easy to see that

$$
\begin{aligned}
& \oplus D_{\#}(X)=C_{\#}(X) \quad \text { (usual chains) } \\
& \Pi D_{\#}(X)=C_{l f}(X) \quad \text { (loc.fin.chains) } \\
& \oplus D^{\#}(X)=C_{c s}(X) \quad \text { (cochains with compact supp) } \\
& \Pi D^{\#}(X)=C^{\#}(X) \quad \text { (usual cochains) }
\end{aligned}
$$

Definition 3.5. A bounded CW complex $X \rightarrow M$ is a simply connected Poincaré duality space if it is 0 and 1 -connected and there is a class $[X] \in H_{n}^{l f}(X ; \mathbb{Z})$ so that

$$
[X] \cap_{-}: D^{\#}(X) \rightarrow D_{n-\#}(X)
$$

is a homotopy equivalence (of chain complexes in $\mathcal{C}_{M}(\mathbb{Z})$ ).

With this definition the elements to develop a simply connected surgery theory are there and this suffices if the reader is mainly interested in applications to resolution of ANR homology manifolds. For other applications, we need to give up the assumption of simply connectedness and work with bounded free G-CW-complexes instead.

Given a ring $R$ and a group $G$ acting on a metric space $M$ by quasi isometries, we define the category $\mathcal{C}_{M, G}(R)$ as follows.

Definition 3.6. An object of $\mathcal{C}_{M, G}(R)$ is a left RG-module $A$ together with a set map $f: A \rightarrow F(M)$, where $F(M)$ is the finite subsets of $M$ such that

(i) $f$ is $G$-equivariant

(ii) $A_{x}=\{a \in A \mid f(a) \subseteq\{x\}\}$ is a finitely generated free sub $R$-module.

(iii) As an R-module $A=\oplus_{x \in M} A_{x}$.

(iv) $f(a+b) \subseteq f(a) \cup f(b)$.

(v) The set $\left\{x \in M \mid A_{x} \neq 0\right\}$ is locally finite.

A morphism $\varphi: A \rightarrow B$ is a morphism of RG-modules so that $\varphi_{n}^{m}: A_{m} \rightarrow B_{n}$ are 0 for $d(m, n)>k$ for some $k=k(\varphi)$.

In case $G$ is the trivial group, $\mathcal{C}_{M, e}(R)$ and $\mathcal{C}_{M}(R)$ are identified by sending an object $A$ in $\mathcal{C}_{M}(R)$ to $\oplus_{x \in M} A_{x}$ together with the map $f: \oplus_{x \in M} A_{x} \rightarrow F(M)$ picking out non-zero coefficients. Similarly when the action of $G$ on $M$ is trivial and $G$ is finite, the categories $\mathcal{C}_{M, G}(R)$ and $\mathcal{C}_{M}(R G)$ may be identified.

If $X \rightarrow M$ is a free bounded G-CW complex, the cellular chains are denoted $D_{\#}(X)$ when thought of as a chain complex in the category $\mathcal{C}_{M, G}(\mathbb{Z})$. In case the action of $G$ on $M$ is trivial this is of course just the chains of the universal cover of $X / G$ in the category $\mathcal{C}_{M}(\mathbb{Z})$ (if $X$ is 1-connected). 
If $R$ is a ring with involution, the category $\mathcal{C}_{M, G}(R)$ has an involution given by $A^{*}=$ $\operatorname{Hom}_{R}^{l f}(A, R)$, the set of locally finite R-homeomorphisms. We define $f^{*}: A^{*} \rightarrow F(M)$ by $f^{*}(\phi)=\left\{x \mid \phi\left(A_{x}\right) \neq 0\right\}$ which is finite by assumption. We define the cellular cochains of $X, D^{\#}(X)$ using this involution, $D^{\#}(X)=\left(D_{\#}(X)\right)^{*}$.

Definition 3.7. A free bounded G-CW complex $X \rightarrow M$ is a Poincaré duality complex if $X \rightarrow M$ is 0 and 1-connected and there is a class $[X] \in H^{l f}(X / G ; C)$ so that a transfer of $[X]$ induces a bounded homotopy equivalence $[X] \cap_{-}: D^{\#}(X) \rightarrow D_{\#}(X)$.

As in the non-equivariant case we have

Theorem 3.8. There is a surgery exact sequence

$$
\rightarrow L_{n+1}\left(\mathcal{C}_{M, G}(\mathbb{Z})\right) \rightarrow \mathcal{S}_{G}^{b}\left(\begin{array}{c}
X \\
\downarrow \\
M
\end{array}\right) \rightarrow[X / G ; F / T O P] \rightarrow L_{n}\left[\mathcal{C}_{M, G}(\mathbb{Z})\right] \rightarrow
$$

Notice that if $G$ is finite, $\mathcal{S}_{G}^{b}\left(\begin{array}{c}X \\ \downarrow \\ M\end{array}\right)$ can be safely replaced by $\mathcal{S}^{b}\left(\begin{array}{c}X / G \\ \downarrow \\ M / G\end{array}\right)$.

\section{Germ methods}

Given a metric space $M$ with an action by $G$ and an equivariant metric subspace $N \subset M$, let us denote the $k$-neighborhood of $N$ by $N^{k}$. We shall develop germ methods "away from $N^{\prime \prime}$.

Definition 4.1. The category $\mathcal{C}_{M, G}^{>N}(R)$ has objects the same as $\mathcal{C}_{M, G}(R)$, but two morphisms $\varphi_{1}$ and $\varphi_{2}: A \rightarrow B$ are identified if there exists $k$ such that $\varphi_{1 y}^{x}=\varphi_{2 y}^{x}$ except on $A_{x}$ where $x$ lies in $N^{k}$.

Using the methods of [44] and [52, 53], the following is easy to see:

Consider $M \cup N \times[0, \infty)$ with metric included from $M \times[0, \infty)$.

Theorem 4.2. The forgetful map (functor!)

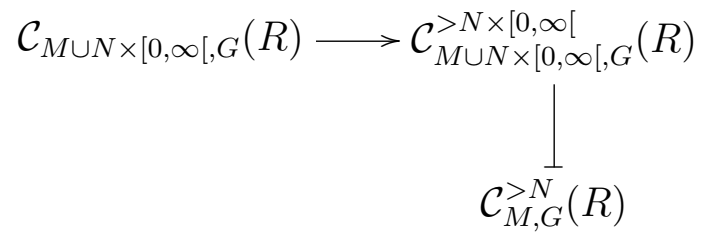

induces isomorphisms on algebraic K-theory and if $R$ is a ring with involution on algebraic L-theory.

Having introduced the category $\mathcal{C}_{M, G}^{>N}(R)$ we may define what we mean by $X \rightarrow M$ being a Poincaré duality complex "away from $N$ ", about a map being a bounded homotopy equivalence "away from $N$ " about a space being -1, 0 or 1-connected "away from $N$ ". We leave this to the reader and satisfy ourselves by the following 
Example 4.3. Let $M$ be a manifold $M \subset S^{N}$, some large $N$. Let $O(M)=\{t \cdot m \mid t \in$ $[0, \infty), m \in M\} \subseteq \mathbb{R}^{N+1}$. Consider $M \times[0, \infty) \rightarrow O(M)$ sending $(m, t)$ to $t \cdot m$. . It is of course a bounded CW complex. It is -1 and 0 -connected but it is 1-connected only "away from 0". The obvious fact that a manifold is locally simply connected translates to $M \times[0, \infty) \rightarrow O(M)$ being 1-connected "away from 0 ".

If $X \rightarrow M$ is a bounded G-Poincaré duality complex, then we have

Theorem 4.4. There is a surgery exact sequence away from $N$

$$
L_{n+1}\left(\mathcal{C}_{M, G}^{>N}(\mathbb{Z})\right) \rightarrow \mathcal{S}^{>N}\left(\begin{array}{c}
X \\
\downarrow \\
M
\end{array}\right) \rightarrow[X, F / T O P]^{>N} \rightarrow L\left(\mathcal{C}_{M, G}^{>N}(\mathbb{Z})\right)
$$

\section{Applichtions of Simply CONNECTED BOUNDED SURGERY}

Consider the surgery exact sequence based on Example 4.3

$$
\rightarrow \mathcal{S}^{>0}\left(\begin{array}{c}
M \times[0, \infty) \\
\downarrow \\
O(M)
\end{array}\right) \rightarrow[M, F / \mathrm{TOP}] \rightarrow L_{n}\left(\mathcal{C}_{O(M)}^{>0}(\mathbb{Z})\right)
$$

according to Theorem $4.2 L_{n}\left(\mathcal{C}_{O(M)}^{>0}(\mathbb{Z})\right) \cong L_{n}\left(\mathcal{C}_{O\left(M^{+}\right)}(\mathbb{Z})\right)$.

Lemma 5.1. $\mathcal{S}_{b}^{>0}\left(\begin{array}{c}M \times[0, \infty) \\ \downarrow \\ O(M)\end{array}\right)=*$.

Proof. This is equivalent to Siebenmann's theorem [54]. It follows that

$$
L_{*}\left(\mathcal{C}_{O\left(M^{+}\right)}(\mathbb{Z})\right) \cong[M, F / \mathrm{TOP}]
$$

for $* \geq M$ but the groups $L_{*}\left(\mathcal{C}_{C\left(M^{+}\right)}(\mathbb{Z})\right)$ are 4-periodic so this identifies (via Poincaré duality) $L_{*}\left(\mathcal{C}_{O\left(M^{+}\right)}(\mathbb{Z})\right)$ with unreduced homology of $M$ with coefficients in the 4-periodic (so non-connective) $\mathbb{L}(\mathbb{Z})$-spectrum, a statement totally analogous to the main theorem in [44].

Remark 5.2. Turning things around, using the algebraic work of Ranicki [52, 53], the above theorem may be used to give a new proof of Siebenmann's theorem.

We now want to apply this for resolution of ANR-homology manifolds [50]. Let $X$ be an ANR-homology manifold. Consider $X \times[0, \infty) \rightarrow O(X)$ away from 0 . Since $X$ is an ANR, this is clearly of the bounded homotopy type of a bounded CW-complex 1-connected away from 0, and satisfies Poincaré duality away from 0 . Assume $X$ has a TOP reduction of the Spivak normal fibre space. The surgery exact sequence is now

$$
\mathcal{S}^{>0}\left(\begin{array}{c}
X \times[0, \infty) \\
\downarrow \\
O(X)
\end{array}\right) \rightarrow[X, F / \mathrm{TOP}] \rightarrow L_{n}\left(\mathcal{C}_{O(X)}^{>0}(\mathbb{Z})\right)
$$

but

$$
L_{N}\left(\mathcal{C}_{O(X)}^{>0}(\mathbb{Z})\right) \cong h_{n}(X, \mathbb{L}(\mathbb{Z})) \stackrel{P . D}{\rightarrow} \cong h^{0}(X, \mathbb{L}(\mathbb{Z}))=[X, F / \mathrm{TOP} \times \mathbb{Z}]
$$


so we see that varying the reduction does not remove the possibility of a $\mathbb{Z}$-obstruction to $\mathcal{S}^{>0}\left(\begin{array}{c}X \times[0, \infty) \\ \downarrow \\ O(X)\end{array}\right)$ being empty. On the other hand, an element in

$\mathcal{S}^{>0}\left(\begin{array}{c}X \times[0, \infty) \\ \downarrow \\ O(X)\end{array}\right)$ produces a resolution of $X$ by Quinn's end theorem [47].

Now let us not assume that $X$ has a TOP reduction. Let $U$ be an open subset of $X$ so that the restriction to $U$ does have a TOP-reduction and $Y=X-U$ in the surgery exact sequence

$$
\mathcal{S}^{>0(Y)}\left(\begin{array}{c}
X \times[0, \infty) \\
\downarrow \\
O(X)
\end{array}\right) \rightarrow[U, F / \mathrm{TOP}] \rightarrow L_{n}\left(\mathcal{C}_{O(X)}^{>O(Y)}(\mathbb{Z})\right) \cong[U, F / \mathrm{TOP} \times \mathbb{Z}]
$$

Once again we may choose the lift so that there is only a $\mathbb{Z}$-obstruction to $\mathcal{S}^{>0(Y)}\left(\begin{array}{c}X \times[0, \infty) \\ \downarrow \\ O(X)\end{array}\right)$ being nonempty.

In other words we have a recipe for choosing the lift on $U$ which is canonical under restriction but that implies

Theorem 5.3. ([22]) An ANR homology manifold has a canonical Top reduction.

Ferry-Pedersen and Daverman, among others, have noticed the following theorem. The pretty geometric proof was shown to us by Daverman.

Theorem 5.4. There is no nonresolvable ANR homology manifold which is homotopy equivalent to $T^{n}$.

Proof. Let $X$ be an ANR homology manifold homotopy equivalent to $T^{n}$. The universal cover $\tilde{X}$ of $X$ can be compactified by a sphere to an ANR homology manifold with a manifold boundary. But homology manifolds with manifold boundaries are resolvable on the boundary, and the obstruction to resolution is local, so $X$ admits a resolution.

Using bounded surgery methods it can be proved that this theorem remains true, if $T^{n}$ is replaced by any other manifold, for which the assembly map is a monomorphism on the fundamental class, e. g. a manifold admitting a map of nonzero degree to the torus [23].

Shmuel Weinberger has pointed out the following direct connection between bounded topology and the Novikov Conjecture. If $f: N \rightarrow M$ is a homotopy equivalence between closed $K(\pi, 1)$ manifolds, then the map $\tilde{f} \times_{\pi} \tilde{f}: \tilde{N} \times_{\pi} \tilde{N} \rightarrow \tilde{M} \times_{\pi} \tilde{M}$ is a map of topological tangent bundles covering $f$. If one can show that the bounded structure set of $\tilde{M}$ is trivial, i. e.

$$
L_{*}\left(\mathcal{C}_{\pi}(\mathbb{Z})\right) \cong h_{*}^{l f}(\widetilde{K}(\pi, 1), G / T o p)
$$

then stably $\tilde{f} \times_{\pi} \tilde{f}$ is fibrewise boundedly homotopic to a homeomorphism and the normal invariant of $f$ vanishes. See [18] and [24] for details. Ferry and Weinberger have recently extended this approach to give a proof of the Novikov conjecture for word hyperbolic groups and for fundamental groups of nonpositively curved polyhedra using bounded methods. The 
argument also shows that if $K$ is an aspherical polyhedral Poincaré duality space in either of these classes, then the Spivak normal bundle of $K$ has a TOP reduction.

In addition, Ferry-Weinberger and Bryant-Mio have recently used bounded methods in constructing examples of nonresolvable ANR homology manifolds. Here is a statement of the main result

Theorem 5.5. $\quad$ (i) ANR homology manifolds homotopy equivalent to a given Poincaré complex $Y$ ( up to s-cobordisms of homology manifolds) are in 1-1 correspondence with what is predicted by the algebraic theory of surgery. In other words, if the total algebraic surgery obstruction of a Poincaré complex $Y$ vanishes, $Y$ is homotopy equivalent to an ANR homology manifold $X$. These are then classified up to s-cobordism by the usual surgery exact sequence with $G / T O P \times \mathbb{Z}$ replacing $G / T O P$.

(ii) It follows that there are ANR homology manifolds not homotopy equivalent to manifolds.

(iii) With the correction of the definition of structure set to include s-cobordism classes of ANR homology manifolds, Siebenmann's periodicity theorem becomes correct.

\section{Functorial properties of Bounded surgery Groups. Mackey properties}

Given a map $M \rightarrow N$ of metric spaces (equivariant metric spaces) which is eventual Lipschitz, there is an induced map

$$
L_{n}\left(\mathcal{C}_{M, G}(R)\right) \rightarrow L_{n}\left(\mathcal{C}_{N, G}(R)\right) .
$$

The geometric interpretation is the following: An element in $L_{n}\left(\mathcal{C}_{M, G}(\mathbb{Z})\right)$ is an obstruction to a -1,0,1-connected G-surgery problem

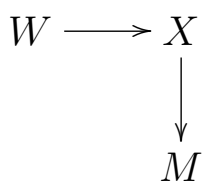

mapping $M$ to $N$ destroys 0,1 and -1-connectedness. This is similar to the situation in classical surgery theory, when trying to interpret a map of fundamental groups geometrically. The vanishing of the surgery obstruction only implies we can do surgery after doing simultaneous surgery on source and target to get a $-1,0$ and 1-connected problem.

The groups $L_{n}\left(\mathcal{C}_{M, G}^{>N}(\mathbb{Z})\right)$ have covariant functorial properties in $M$, contravariant functorial properties in $N$, covariant functorial properties in $\mathbb{Z}$, and finally there is the variable $G$. Let $G$ be a finite group.

Theorem 6.1. ([30]) The functors $L_{n}\left(\mathcal{C}_{M,-}^{>N}(R)\right)$ are Mackey functors on the category of subgroups of $G$.

This theorem is a strong computational tool which is used in the next section. 


\section{Applications to Group Actions.}

In this section we will describe some of the results of [30] and state some open problems.

Question 7.1. (C.T.C. Wall [56], F.T. Farrell)

What are the possible finite subgroups of a group $\Gamma$ which acts smoothly, freely and properly discontinuously on some product $S^{n} \times R^{m}$ with compact quotient?

This question is motivated by the compact space form problem. Recall that if a finite group $G$ acts freely and simplicially on a complex homotopy equivalent to a sphere $S^{n}$ then $S^{n}$ has periodic Tate cohomology with period dividing $n+1$. This is an obvious necessary condition for 7.1. In addition, we wish to assume that our groups are finitely generated and have finite virtual cohomological dimension $(\operatorname{vcd}(\Gamma)<\infty)$. It follows that we will be working with groups $\Gamma$ having periodic Farrell cohomology [17].

Properly discontinuous group actions on $S^{n} \times R^{m}$ arise from pseudo-Riemannian space forms (see [61], Ch. 11 and [37]). If $V=R^{n+m+1}$ we can define the quadratic form

$$
Q\left(x_{1}, \ldots, x_{n+1}, y_{1}, \ldots, y_{m}\right)=\sum_{i=1}^{n+1} x_{i}^{2}-\sum_{i=1}^{m} y_{i}^{2},
$$

and let $S^{n, m}$ denote the component of $\{v \in V \mid Q(v)=1\}$ containing the point $p_{0}=$ $(1,0, \ldots, 0)$. Let $O(n+1, m)$ denote the subgroup of the orthogonal group $O(Q)$ preserving $S^{n, m}$ and observe that $S^{n, m} \approx O(n+1, m) / O(n, m)$ where $O(n, m)$ is the isotropy subgroup at $p_{0}$. A classical problem in geometry is to classify subgroups $\Gamma \subset O(n+1, m)$ which act freely and properly discontinuously on $S^{n, m}$. Note that since the isotropy group of $p_{0}$ is non-compact, a discrete subgroup of $O(n+1, m)$ does not automatically act properly discontinuously. This problem contains the spherical (resp. hyperbolic) space form problems as the special cases $m=0$ (resp. $n=0$ ). The intermediate cases, where $Q$ is indefinite, are called pseudo-Riemannian space forms even when $\Gamma$ does not act freely. Since $S^{n, m} \approx S^{n} \times R^{m}$ for $n>0$ we recover the situation of (7.1) whenever $\Gamma$ acts both freely and co-compactly.

The pseudo-Riemannian space forms are somewhat restricted from the topological point of view (see [37] for proofs and references).

7.2. Restrictions on Classical Space Forms

(i) If $n \geq m$ then only finite subgroups of $\Gamma$ can act properly discontinuously on $S^{n, m}$.

(ii) If $n, m$ odd, then there are no compact examples $\Gamma \backslash S^{n, m}$.

(iii) If $\operatorname{vcd}(\Gamma)<\infty$ then $\operatorname{vcd}(\Gamma) \leq m$ with equality if and only if $\Gamma \backslash S^{n, m}$ is compact.

(iv) If $G \subset \Gamma$ is a finite subgroup; then $G$ acts freely and orthogonally on $S^{n}$.

(v) If $n>0, m>0$ then $\Gamma$ cannot be an arithmetic subgroup of $O(n+1, m)$.

(vi) If $n+1<m$ there exist subgroups of $O(n+1, m)$ isomorphic to fundamental groups of orientable surfaces which act properly discontinuously on $S^{n, m}$.

As a test case for Question (7.1), F.T. Farrell suggested that we consider the groups $\Gamma=\mathbb{Z}^{k} \rtimes_{\alpha} D_{p}$ where $D_{p}$ denotes a finite dihedral group of order $2 p, p$ prime, and $\alpha$ a 
homomorphism giving the semi-direct product. Note that the dihedral groups $D_{p}$ are the simplest groups which have periodic Tate cohomology, but do not act freely and orthogonally on a sphere. These groups therefore have periodic Farrell cohomology but by (7.2) (iv) do not occur for classical space forms. On the other hand, Connolly and Prassidis [15] proved that any countable group $\Gamma$ with $\operatorname{vcd}(\Gamma)<\infty$ and periodic Farrell cohomology acts freely and properly discontinuously on some product $S^{n} \times R^{m}$, but their construction does not produce actions with compact quotient.

Our approach to answering (7.1) for the groups $\Gamma=\mathbb{Z}^{k} \rtimes_{\alpha} D_{p}$ is to relate smooth, free, co-compact $\Gamma$ actions on $S^{n} \times R^{m}$ to topological $D_{p}$ actions on $S^{n+m}$ with an invariant $S^{m-1} \subset S^{n+m}$ via a compactification process. In the following statements we let $R_{-}$denote the non-trivial one-dimensional representation of $D_{p}$.

Theorem 7.3. ([30]) Let $V$ be a linear representation of the dihedral group $G=D_{p}, p$ an odd prime. Then there is a topological action of $G$ on a sphere, free off a standard proper subsphere, and given by the unit sphere $S(V)$ on the subsphere, if and only if the representation $V$ has at least two $R_{-}$factors.

Theorem 7.4. ([30]) The group $\Gamma=\mathbb{Z}^{k} \rtimes_{\alpha} D_{p}$ acts freely and properly discontinuously on $S^{n} \times R^{m}$ with compact quotient if and only if $n \equiv 3(\bmod 4), m=k$ and $\alpha$ considered as a real representation has at least two $R_{-}$factors.

Our non-existence result concern topological actions, but the actions constructed in Theorem 7.4 are smooth. The lowest dimensional example is a co-compact action on $S^{3} \times R^{2}$. We begin with a sketch of the argument for Theorem 7.3.

If a finite group $G$ acts on $S^{n+k}$ free off a standard proper subsphere $S^{k-1}$, then the homotopy type of the free part is determined by the quotient space $X=\left(S^{n+k}-S^{k-1}\right) / G$. Bounded surgery provides a natural way to study such actions on spheres, by considering the free part as an element of a bounded structure set. Let $\alpha: G \rightarrow O(k)$ determine the $G$-action restricted to $S^{k-1}$. More precisely, we have a bijective correspondence

$$
\mathcal{S}^{b}\left(\begin{array}{c}
\tilde{X} \times G R^{k} \\
\downarrow \\
O\left(S^{k-1} / G\right)
\end{array}\right) \leftrightarrow\left\{\begin{array}{c}
\text { conjugacy classes of group actions } \\
\text { on } S^{n+k}, \text { given by } \alpha \text { on } S^{k-1} \text { and } \\
\text { with }\left(S^{n+k}-S^{k-1}\right) / G \simeq X^{n}
\end{array}\right\}
$$

In this formula, $X^{n}$ can be any finite $n$-dimensional Swan complex (i. e. $\pi_{1}(X) \cong G$ and universal covering $\tilde{X} \simeq S^{n}$ ) and $G$ acts on $R^{k}=O\left(S^{k-1}\right)$ by the cone of the action $\alpha$.

Now the left-hand side can be studied by means of the (bounded) surgery exact sequence. In the case $G=D_{p}$ we start with a finite Swan complex $X$, and use the natural transformation of surgery exact sequences associated to the product pairings:

$$
\mathcal{C}_{U, G}(R) \times \mathcal{G}_{V, G}(R) \rightarrow \mathcal{C}_{U \oplus V, G}(R),
$$

for $R=\mathbb{Z}, \hat{\mathbb{Z}}_{2}$ and $U, V$ real representations of $G$. In the case $R=\mathbb{Z}, U=\{0\}$ and $V$ the representation given by $\alpha$ we get a transfer map $\operatorname{trf}_{V}$ which induces the identity on the 
normal invariant term:

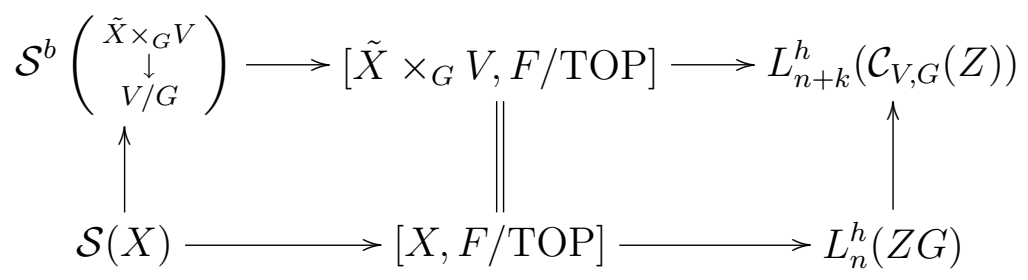

If $f: M^{n} \rightarrow X^{n}$ denotes a degree 1 normal map, then Milnor [39] showed in effect that the surgery obstruction $\sigma=\sigma(f) \in L_{n}^{h}(\mathbb{Z} G)$ is non-zero. We use the Mackey properties of the bounded surgery groups and knowledge of the 2-adic behavior of $\sigma$ to conclude that $\operatorname{trf}_{V}(\sigma)=0$ if and only if $V$ contains at least two $R_{-}$factors.

For the non-existence part of Theorem 7.4 we show that whenever $\Gamma=\mathbb{Z}^{k} \rtimes_{\alpha} G$ acts freely and co-compact on $S^{n} \times R^{k}$ then $G$ acts on $S^{n+k}$ as above. We consider $S^{n+k}=$ $\left(S^{n} \times R^{k}\right) \cup S^{k-1}$ and use the compact quotient $\left(S^{n} \times R^{k}\right) / \Gamma$ to perform a $G$-equivariant compactification of the $\Gamma$ action restricted to $G$. We adjoin an $S^{k-1}$ at $\infty$ equipped with the a $G$-action induced by the associated orthogonal representation to $\alpha: G \rightarrow G L_{k}(\mathbb{Z})$.

The existence part of Theorem B is proved by an explicit "blocked surgery" argument starting with the compact surgery problem $\tilde{f} \times_{G} 1:(\tilde{M} \rightarrow \tilde{X}) \times_{G} T^{k}$, where $G$ acts on $T^{k}$ via $\alpha$. If the associated representation to $\alpha$ has at least two $R_{-}$factors, we show that $\sigma\left(\tilde{f} \times_{G} 1\right)=0$. The group $\Gamma=\mathbb{Z}^{k} \rtimes_{\alpha} G$ then acts freely and co-compactly on the universal covering, which can be arranged to be diffeomorphic to $S^{n} \times R^{k}$.

\subsection{Extensions}

Our methods have been extended both to construct more examples of groups acting freely and co-compactly on $S^{n} \times R^{k}$ and to show that some other groups do not act.

The action on $T^{2}$ given by complex conjugation can be pictured as rotating a standard picture of the torus in $R^{3}$ visibly having exactly four fixed points. This picture can be used in the blocked surgery computation of the surgery obstruction in $L^{h}\left(\mathbb{Z}\left(G \times{ }_{-} \mathbb{Z}^{2}\right)\right.$ which shows that a certain surgery obstruction vanishes, and thus implies the existence of an action of $G \times \mathbb{Z}^{2}$ on $S^{n} \times \mathbb{R}^{2}$. Chris Stark observed that there is a similar action on a surface $F$ of genus $g$ with $4+2 g$ fixed points, and thus essentially the same surgery computation implies

Theorem 7.6. ([55]) There is a proper discontinuous, cocompact free action of action of $G \times \pi_{1}(F)$ on $S^{n} \times \mathbb{R}^{2}$.

The results have also been extended in the direction of non-existence. Doug Anderson and Frank Connolly have proved the following extension.

Theorem 7.7. ([1]) Let $\Gamma$ be a group that acts on a complete non-positively curved manifold, such that restricted to a cofinal subgroup, the action is proper discontinuous, free and cocompact. Assume $\Gamma$ contains a dihedral subgroup $D_{p}$. If the difference between the virtual 
cohomological dimension of the centralizer of $D_{p}$ in $\Gamma$ and the virtual cohomological dimension of the centralizer of $C_{p}$ in $\Gamma$ is less than 2, then $\Gamma$ can not act properly discontinuously, freely, cocompactly on a sphere crossed with euclidean space.

This theorem is proved by showing that the existence of such an action, by a compactification argument similar to the one given in [30] implies the existence of a $D_{p}$-action on a sphere $S^{n+k}$ free off a subsphere $S^{k-1}$, and given by a representation $\alpha$ with less than 2 $R_{-}$-summands on $S^{k-1}$ thus contradicting Theorem 7.3.

7.8. Open problems

(i) Formulate a reasonable conjecture for the answer to (7.1) if $\Gamma$ is a crystallographic group: an extension $1 \rightarrow \Gamma_{0} \rightarrow \Gamma \rightarrow G \rightarrow 1$, where $\Gamma_{0} \cong \mathbb{Z}^{k}$ and the finite group $G$ acts faithfully on $\Gamma_{0}$.

(ii) If $G$ is finite and $\alpha: G \rightarrow G L_{k}(\mathbb{Z})$ induces the real representation $V \cong \mathbb{R}^{k}$, study the transfer map $\operatorname{trf}_{V}$ on L-theory. Is there a (split) injection

$$
L_{n+k}^{h}\left(\mathcal{C}_{V, G}(\mathbb{Z})\right) \hookrightarrow L_{n+k}^{h}\left(\mathbb{Z}\left[\mathbb{Z}^{k} \rtimes_{\alpha} G\right]\right) ?
$$

Notice that in the special case when $\alpha$ is trivial, the answer is "yes", since

$$
L_{n+k}^{h}\left(\mathcal{C}_{R^{k}, G}(\mathbb{Z})\right) \cong L_{n}^{\langle 1-k\rangle}(\mathbb{Z} G)
$$

and our transfer map corresponds to "crossing with $T^{k}$ " [51]. In this special case, the bounded surgery group is also isomorphic to the subgroup of $L_{n+k}^{h}\left(\mathbb{Z}\left[\mathbb{Z}^{k} \times G\right]\right)$ invariant under restrictions to a cofinal family $\left\{F \times G: F \subseteq \mathbb{Z}^{k}\right.$ of finite index $\}$. Can this be generalized to the cases when $\alpha$ is non-trivial ?

\subsection{Non-linear Similarity}

Two real representations $\rho_{1}, \rho_{2}$ of a finite group $G$ are topologically equivalent $\left(\rho_{1} \sim_{t} \rho_{2}\right)$ if there exists a homeomorphism $h: V_{1} \rightarrow V_{2}$, where $V_{1}, V_{2}$ are the associated representation spaces. Let $R_{\mathrm{TOP}}(G)$ be the Grothendieck group of $G$-homeomorphism classes of real representations under direct sum. Thus $R_{\mathrm{TOP}}(G)$ is the quotient of $R O(G)$ obtained by identifying two real representations $\rho_{1}, \rho_{2}$ if $\rho_{1}+\theta \sim_{t} \rho_{2}+\theta$ for some representation $\theta$. This equivalence relation ("non-linear similarity") and $R_{\mathrm{TOP}}(G)$ have been extensively studied [6], [7], [32], [38].

In recent work [31] we show that bounded surgery methods are useful for (i) obtaining information about $R_{\mathrm{TOP}}(G)$ and (ii) constructing further examples of non-linear similarities, e. g. for non-cyclic groups.

The basic idea can be seen by reviewing the construction in [6]. Let $G=\mathbb{Z}_{4 q}$ and suppose that $\rho_{1}, \rho_{2}$ are free $(n+1)$-dimensional representations of $G$ (i. e. $G$ acts freely on the representation spaces $V_{1}, V_{2}$ away from 0 ) such that (i) $\operatorname{Res}_{H}\left(\rho_{1}\right) \cong \operatorname{Res}_{H}\left(\rho_{2}\right)$ when $H=$ $\mathbb{Z}_{2 q} \subset G$, and (ii) $\rho_{1}$ and $\rho_{2}$ are $G$-homotopy equivalent, say by $f: S\left(V_{1}\right) / G \rightarrow S\left(V_{2}\right) / G$. Now $\left(S\left(V_{1}\right) / G, f\right)$ can be considered as an element in $\mathcal{S}\left(S\left(V_{2}\right) / G\right)$, and it follows that there 
exists an element $\tau(f) \in L_{n+1}^{h}\left(\mathbb{Z}\left[\mathbb{Z}_{4 q}\right]\right)$ such that $\tau(f) *\left(S\left(V_{2}\right) / G, i d\right)=\left(S\left(V_{1}\right) / G, f\right)$ under the usual action. A "bounded surgery" explanation for the rest of the construction is given by:

Theorem 7.10. ([31]) Let $G$ be a finite group and $V_{1}, V_{2}$ and $W$ be real representation spaces of $G$. Suppose that there exists a homotopy equivalence $f: S\left(V_{1}\right) / G \rightarrow S\left(V_{2}\right) / G$ and an element $\tau(f) \in L_{n+1}^{h}(\mathbb{Z} G), n+1=\operatorname{dim} V_{1}=\operatorname{dim} V_{2}$, such that $\tau(f) *\left(S\left(V_{2}\right) / G, i d\right)=$ $\left(S\left(V_{1}\right) / G, f\right)$. Then there exists a $G$-h-cobordism between $S\left(V_{1} \oplus W\right)$ and $S\left(V_{2} \oplus W\right.$ provided that $\operatorname{trf}_{W}(\tau(f))=0$ under the transfer $\operatorname{trf}_{W}: L_{n+1}^{h}(\mathbb{Z} G) \rightarrow L_{n+k}^{h}\left(\mathcal{C}_{W, G}(\mathbb{Z})\right), k=\operatorname{dim} W$.

Corollary 7.11. If $\rho_{1}, \rho_{2}$ and $\theta$ are the associated $G$-representations to $V_{1}, V_{2}$, and $W$, then $\operatorname{trf}_{W}(\tau(f))=0$ implies that $\rho_{1}+\theta+\epsilon \sim_{t} \rho_{2}+\theta+\epsilon$, where $\epsilon$ denotes the trivial 1-dimensional representation.

This result combined with our methods for computing such bounded surgery transfers leads to the applications mentioned above. The detailed results will appear in [31].

\section{Estimated STABILITy AND VANiSHING THEOREMS}

Following [11] and [47], we define

Definition 8.1. A geometric $\mathbb{Z} \pi$-module on $K$ is an object $A$ in $\mathcal{C}_{K}(\mathbb{Z} \pi)$ such that each $A_{x}$ has a preferred basis. A deformation is a composable string $e_{1}, \ldots, e_{n}$ of elementary isomorphisms. The bound of a deformation is the $\max$ of bound $\left(e_{i} \circ \cdots \circ e_{1}\right), i \leq n$. A $\delta$-isomorphism is an isomorphism $\alpha: A \rightarrow B$ such that bound $(\alpha)$ and bound $\left(\alpha^{-1}\right)$ are both less than $\delta$.

Here is Quinn's Stability Theorem. For clarity, we state the theorem for finite polyhedra. The generalization to locally compact ANR's is not difficult.

Theorem 8.2. ([48], p. 381) Suppose that $K$ is a finite polyhedron. Then there is an $\epsilon_{0}>0$ such that

(i) for any $\epsilon, 0<\epsilon<\epsilon_{0}$ there is a $\delta>0$ such that $\epsilon$-deformation is an equivalence relation on $\delta$-isomorphisms of geometric $\mathbb{Z} \pi$ modules for any group $\pi$ and

(ii) the set of equivalence classes form an abelian group, which is naturally isomorphic to the inverse limit of such groups as $\epsilon \rightarrow 0$.

Quinn's proof is a torus argument. While this proof is constructive in principle, extracting explicit bounds would be painful. Working directly with bounded topology avoids the torus and makes the argument remarkably concrete. Here is our theorem.

Theorem 8.3. ([23]) Let $K$ be a cubical subcomplex of the boundary of the unit ball in $\mathbb{R}^{n}$. If $\alpha: A \rightarrow A$ is an automorphism in $\mathcal{C}_{K}(R)$ such that $\alpha$ and $\alpha^{-1}$ are bounded by $\delta>0$ in the max metric, $0<\delta<6^{-(\operatorname{dim} K)}$, then for each $\eta>0$ there is an object $B$ in $\mathcal{C}_{K}(R)$ and an automorphism $\beta: A \oplus B \rightarrow A \oplus B$ such that 
(i) $\beta$ and $\beta^{-1}$ are bounded by $\eta$.

(ii) $\beta=\prod e_{i} \cdot(\alpha \oplus i d)$.

(iii) There are no more than $12 \mathrm{dim} K+18$ terms in the product.

(iv) The bound of each $e_{i}$ is less than $6^{(\operatorname{dim} K)} \delta$.

This follows easily from the next theorem, which shows that sufficiently small automorphisms which are trivial inside of the unit cube can be deformed to the identity.

Theorem 8.4. ([23]) Let $K$ be a cubical subcomplex of the boundary of the unit ball in $\mathbb{R}^{n}$. If $\alpha$ is an automorphism in $\mathcal{C}_{K}(R)$ such that $\alpha$ and $\alpha^{-1}$ are bounded by $\delta<6^{-(\operatorname{dim} K)}$ in the max metric, then there exist an object $B$ in $\mathcal{C}_{O(K)}$, and a product $\prod e_{i}$ of elementary automorphisms of $A \oplus B$ in the category $\mathcal{C}_{O(K)}(R)$ such that :

(i) $B_{x}=0$ for $x \leq 1$.

(ii) $\prod e_{i}=\alpha \oplus i d$.

(iii) Each $e_{i}$ is bounded by $6^{(\operatorname{dim} K)} \delta$.

(iv) The number of elementary automorphisms needed is $\leq 12(\operatorname{dim} K+1)$.

Proof of Theorem 8.3. Let $\alpha: A \rightarrow A$ be an automorphism as in the statement of the theorem. Theorem 8.4 guarantees that $\alpha \oplus \mathrm{id}=\prod_{i=1}^{M} e_{i}$ where we set $M=12(\operatorname{dim} \mathrm{K}+1)$.

Let $L>0$ be large and consider the elementary automorphisms $\bar{e}_{i}$ obtained by setting the $e_{i}$ 's equal to the identity outside of the cube $\|x\| \leq L$.

The product $\prod \bar{e}_{i}$ is equal to $\alpha$ on the unit cube and is equal to some $\bar{\beta}$ on a band near $\|x\|=L$. The product is the identity elsewhere. Projecting back to $\|x\|=1$ gives a deformation from $\alpha$ to the image $\beta$ of $\bar{\beta}$, where bound $(\beta)$ is approximately $\frac{1}{L} \sum$ bound $\left(e_{i}\right)$. Since $L$ may be taken arbitrarily large, this completes the proof.

The proof of Theorem 8.4 is an inductive use of the Eilenberg swindle. The reader is referred to [23] for details. Our methods also show that the stabilized $\epsilon$-Whitehead group described in part (ii) of Quinn's Stability Theorem is naturally isomorphic to $K_{2}\left(C_{O_{\left(K^{+}\right)}}(R)\right)$, where $K^{+}$is the union of $K$ and a disjoint basepoint. Combining this with the PedersenWeibel calculation of Theorem 3.2 gives a rather constructive algebraic proof of the $K$-theory $\alpha$-approximation theorems of [19], [47], [48]. Again, the reader is referred to [23] for details.

\section{Counting simple homotopy types in Gromov-Hausdorff space}

Gromov has introduced a complete metric on the set of isometry classes of compact metric spaces. Here is the definition.

Definition 9.1. If $Z$ is a compact metric space and $X$ and $Y$ are closed subsets of $Z$, then the Hausdorff distance from $X$ to $Y$ in $Z$ is

$$
d_{Z}^{H}(X, Y)=\inf \left\{\epsilon>0 \mid X \subset N_{\epsilon}(Y) \text { and } Y \subset N_{\epsilon}(X)\right\} .
$$


Here, $N_{\epsilon}(X)$ denotes the set of points in $Z$ whose distance from $X$ is less than $\epsilon$. The Gromov-Hausdorff distance from $X$ to $Y$ is

$$
d_{G}(X, Y)=\inf _{Z}\left\{d_{Z}^{H}(X, Y) \mid X \text { and } Y \text { are embedded isometrically in } Z\right\} .
$$

Let $\mathcal{C M}$ denote the set of isometry classes of compact Hausdorff spaces with the GromovHausdorff metric.

Definition 9.2. (i) A function $\rho:[0, R) \rightarrow[0, \infty)$ with $\rho(0)=0$ is a contractibility function if $\rho$ is continuous at 0 and $\rho(t) \geq t$ for all $t$.

(ii) A compact metric space $X$ is locally contractible with contractibility function $\rho$ if for each $r<R$, the ball $B_{r}(x)$ contracts to a point in $B_{\rho(r)}(x)$.

(iii) A subset $\mathcal{S} \subset \mathcal{C M}$ is said to be precompact if $\mathcal{S}$ has compact closure in $\mathcal{C M}$. Since $\mathcal{C M}$ is complete, $\mathcal{S}$ is precompact if and only if it has a finite cover by $\epsilon$-balls for each $\epsilon$.

Let $\mathcal{M}(\rho, n)$ denote the subset of $\mathcal{C} \mathcal{M}$ consisting of isometry classes of compact metric spaces with Lebesgue covering dimension $\leq n$ which have contractibility function $\rho$. This class of spaces arises naturally in geometry. See [26], [27, 28].

Definition 9.3. If $X$ is a compact metric space, we will say that $N:(0, \beta) \rightarrow(0, \infty)$ is a capacity function for $X$ if for each $\epsilon \in(0, \beta), X$ contains no more than $N(\epsilon)$ disjoint $\epsilon$-balls.

Theorem 9.4. (Gromov Compactness Theorem[46]) Let $\mathcal{S} \subset \mathcal{C M}$ be a collection of compact metric spaces. The following are equivalent.

(i) $\mathcal{S}$ is precompact.

(ii) There is a function $N:(0, \beta) \rightarrow(0, \infty)$ which is a capacity function for every $X \in \mathcal{S}$.

The estimated vanishing results of Section 8 can be used to prove:

Theorem 9.5. ([21]) Any precompact subset of $\mathcal{M}(\rho, n)$ contains only finitely many simple homotopy types.

In fact, the number of simple homotopy types in a precompact class with contractibility function $\rho$ can be estimated in terms of $\rho$ and a capacity function or, better yet, a "packing function" which says how many disjoint $\epsilon$-balls can be in a $6 \epsilon$-ball. This can be used, for instance, to estimate the number of simple homotopy types in the class of Riemannian manifolds with a lower bound on curvature, an upper bound on diameter, and a lower bound on volume. For details, the reader is referred to [21], [45], [46],[26], [27], and [25]. Ferry has recently extended this argument to prove the analogous result for homeomorphism types:

Theorem 9.6. Every precompact subset of $\mathcal{M}(\rho, n) \cap\{$ closed $n$-manifolds $\}$ contain only finitely many homeomorphism types. 


\section{REFERENCES}

1. D. R. Anderson and F. X. Connolly, Finiteness obstructions and cocompact actions on $S^{m} \times \mathbf{R}^{n}$, Comment. Math. Helv. 68 (1993), 85-110.

2. D. R. Anderson and W-C. Hsiang, The functors $K_{-i}$ and pseudoisotopies of polyhedra, Ann. of Math. (2) 105 (1977), 201-233.

3. D. R. Anderson and H. J. Munkholm, Foundations of Boundedly Controlled Algebraic and Geometric Topology, Lecture Notes in Mathematics, vol. 1323, Springer, 1988.

4. D. R. Anderson and E. K. Pedersen, Semifree topological actions of finite groups on spheres, Math. Ann. 265 (1983), 23-44.

5. M. Brown and H. Gluck, Stable structures on manifolds:I-III. Homeomorphisms of $S^{n}$, Ann. of Math. (2) 79 (1964), 1-58.

6. S. E. Cappell and J. L. Shaneson, Non-linear similarity, Ann. of Math. (2) 113 (1981), 315-355.

7. S. E. Cappell, J. L. Shaneson, M. Steinberger, and J. West, Non-linear similarity begins in dimension six, J. Amer. Math. Soc. 111 (1989), 717-752.

8. G. Carlsson, Bounded K-theory and the assembly map in algebraic K-theory, Novikov Conjectures, Rigidity and Index Theorems Vol. 2, (Oberwolfach, 1993), London Math. Soc. Lecture Notes, vol. 227, Cambridge Univ. Press, Cambridge, 1995, pp. 5-127.

9. T. A. Chapman, Topological invariance of Whitehead torsion, J. Amer. Math. Soc. 96 (1974), $488-497$.

10. __ Approximation results in Topological Manifolds, Memoirs, vol. 251, Amer. Math. Soc., Providence, RI, 1981.

11. _ Controlled Simple Homotopy Theory and Applications, Lecture Notes in Mathematics, vol. 1009, Springer, 1983.

12. T. A. Chapman and S. C. Ferry, Approximating homotopy equivalences by homeomorphisms, Amer. J. Math. 101 (1979), 583-607.

13. E. H. Connell, Approximating stable homeomorphisms by piecewise linear ones, Ann. of Math. (2) 78 (1963), 326-338.

14. E. H. Connell and J. Hollingsworth, Geometric groups and Whitehead torsion, Trans. Amer. Math. Soc. 140 (1969), 161-181.

15. F. Connolly and S. Prassidis, Groups which act freely on $R^{m} \times S^{n-1}$, Topology 28 (1989), 133-148.

16. R. D. Edwards and R. C. Kirby, Deformations of spaces of embeddings, Ann. of Math. (2) 93 (1971), 63-88.

17. F. T. Farrell, An extension of Tate cohomology to a class of infinite groups, J. Pure Appl. Algebra 10 (1977), 153-161.

18. F. T. Farrell and W. C. Hsiang, The topological-euclidean space form problem, Invent. Math. 45 (1978), 181-192.

19. S. C. Ferry, The homeomorphism group of a compact Q-manifold is an ANR, Ann. of Math. (2) 106 (1977), 101-120.

20. __ Homotoping €-maps by homeomorphisms, Amer. J. Math. 101 (1979), 567-582.

21. __ Counting simple homotopy types in Gromov-Hausdorff space, preprint, 1991.

22. S. C. Ferry and E. K. Pedersen, Controlled algebraic K-theory, 1987.

23. __ Epsilon surgery Theory, Novikov Conjectures, Rigidity and Index Theorems Vol. 2, (Oberwolfach, 1993), London Math. Soc. Lecture Notes, vol. 227, Cambridge Univ. Press, Cambridge, 1995, pp. 167-226.

24. S. C. Ferry and S. Weinberger, Curvature, tangentiality, and controlled topology, Invent. Math. 105 (1991), 401-414. 
25. K. Grove, Metric differential geometry, Differential Geometry (Lyngby, 1985), Lecture Notes in Mathematics, vol. 1263, Springer, Berlin, 1987, pp. 171-227.

26. K. Grove and P. Petersen, Bounding homotopy types by geometry, Ann. of Math. (2) 128 (1988), 195-206.

27. K. Grove, P. Petersen, and J. Wu, Geometric finiteness theorems in controlled topology, Invent. Math. 99 (1990), 205-213.

28. __ Erratum: Geometric finiteness theorems via controlled topology, Invent. Math. 104 (1991), 221-222.

29. I. Hambleton and I. Madsen, Actions of finite groups on $R^{n+k}$ with fixed set $R^{k}$, Canad. J. Math. 38 (1986), 781-860.

30. I. Hambleton and E. K. Pedersen, Bounded surgery and dihedral group actions on spheres, J. Amer. Math. Soc. 4 (1991), 105-126.

31. , Non-linear similarity revisited, Prospects in Topology: (Princeton, NJ, 1994), Annals of Mathematics Studies, vol. 138, Princeton Univ. Press, Princeton, NJ, 1995, pp. 157-174.

32. W-C. Hsiang and W. Pardon, When are topologically equivalent representations linearly equivalent, Invent. Math. 68 (1982), 275-316.

33. C. B. Hughes, Approximate fibrations and bundle maps on Hilbert cube manifolds, Topology Appl. 15 (1983), 159-172.

34. , Approximate fibrations on topological manifolds, Michigan Math. J. 32 (1985), 167-183.

35. C. B. Hughes, L. R. Taylor, and B. Williams, Controlled surgery over manifolds, In preparation.

36. R. Kirby and L. Siebenmann, Foundational Essays on Topological Manifolds, Smoothings and Triangulations, Annals of Mathematics Studies, vol. 88, Princeton Univ. Press, 1977.

37. R. S. Kulkarni, Proper actions and pseudo-Riemannian space forms, Adv. in Math. 40 (1981), 10-51.

38. I. Madsen and M. Rothenberg, On the classification of G-spheres I: equivariant transversality, Acta Math. 160 (1988), 65-104.

39. J. Milnor, Groups which act on $S^{n}$ without fixed points, Amer. J. Math. 79 (1957), 623-630.

40. E. K. Pedersen, $K_{-i}$-invariants of chain complexes, Topology (Leningrad, 1982), Lecture Notes in Mathematics, vol. 1060, Springer, Berlin, 1984, pp. 174-186.

41. __ On the $K_{-i}$ functors, J. Algebra 90 (1984), 461-475.

42. _ Bounded and continuous control, Novikov Conjectures, Rigidity and Index Theorems Vol. 2, (Oberwolfach, 1993), London Math. Soc. Lecture Notes, vol. 227, Cambridge Univ. Press, Cambridge, 1995, pp. 277-284.

43. E. K. Pedersen and C. Weibel, A nonconnective delooping of algebraic K-theory, Algebraic and Geometric Topology, (Rutgers, 1983), Lecture Notes in Mathematics, vol. 1126, Springer, Berlin, 1985, pp. 166-181.

44. __ K-theory homology of spaces, Algebraic Topology, (Arcata, 1986), Lecture Notes in Mathematics, vol. 1370, Springer, Berlin, 1989, pp. 346-361.

45. P. Petersen, A finiteness theorem for metric spaces, J. Differential Geom. 31 (1990), 387-395.

46. Gromov-Hausdorff convergence of metric spaces, Differential Geometry: Riemannian Geometry, (Los Angeles, CA, 1990), Proc. Symp. Pure. Math., vol. 54, Amer. Math. Soc., Providence, RI, 1993, pp. 489-504.

47. F. Quinn, Ends of maps, I, Ann. of Math. (2) 110 (1979), 275-331.

48. _ Ends of maps, II, Invent. Math. 68 (1982), 353-424.

49. An obstruction to the resolution of homology manifolds, Michigan Math. J. 301 (1987), 285-292.

50. , Resolutions of homology manifolds, and the topological characterization of manifolds, Invent. Math. 72 (1987), 267-284.

51. A. A. Ranicki, Algebraic L-theory II, Laurent extensions, Proc. London Math. Soc. (3) 27 (1973), 126158 .

52. _ Additive L-theory, K-theory 3 (1989), 163-195. 
53. _ Lower K- and L-theory, London Math. Soc. Lecture Notes, vol. 178, Cambridge Univ. Press, 1992.

54. L. C. Siebenmann, Approximating cellular maps by homeomorphisms, Topology 11 (1972), 271-294.

55. C. Stark, Groups acting on $S^{k} \times R^{n}$ : generalisations of a construction of Hambleton and Pedersen, K-theory 5 (1992), 333-354.

56. C. T. C. Wall, Periodic projective resolutions, Proc. London Math. Soc. (3) 39 (1979), 509-553.

57. S. Weinberger, The topological Classification of Stratified Spaces, Chicago Lecture Notes in Mathematics, University of Chicago Press, Chicago Ill., 1994.

58. M. Weiss and B. Williams, Automorphisms of manifolds and algebraic K-theory I, K-theory 1 (1988), 575-626.

59. __ Automorphisms of manifolds and algebraic K-theory II, J. Pure Appl. Algebra 62 (1989), 47-107.

60. J. West, Mapping Hilbert cube manifolds to ANR's: A solution to a conjecture of Borsuk, Ann. of Math. (2) 106 (1977), 1-18.

61. J. A. Wolf, Spaces of Constant Curvature, McGraw-Hill, New York, 1967.

SUNY AT BINGHAMTON

McMaster University

SUNY AT BINGHAMTON 